\title{
Wholebody simulator connected with lung simulator for educational purposes
}

\author{
Martin Rozanek, Jan Suchomel, Petr Kudrna, Hana Hejnalova \\ Department of biomedical technology \\ Czech Technical University in Prague \\ Kladno, Czech Republic \\ rozanek@fbmi.cvut.cz
}

\begin{abstract}
The modern trend is to include the novel equipment into the education. The ASL 5000 is a precise simulator of lung mechanics with possible settings of a large amount of intrapulmonary parameters. The ECS simulator can simulate gas diffusion from the lungs to the blood and vice versa, and computes blood $\mathrm{pH}$, partial pressures of blood gases, oxygen saturation etc. This study describes a connection between these two simulators with regard to the optimal use of the properties of both simulators. This connection enables monitoring of lung mechanics and gas diffusion at the same time. The aim of the study is to describe the connection of the systems for the education of the practically oriented students.
\end{abstract}

Keywords-whole-body simulator, lung simulator, respiratory mechanics, spontaneous breathing, artificial lung ventilation

\section{INTRODUCTION}

Use of the mannequins becomes very popular in the all areas of education, mainly of the medical stuff. Mannequin ECS (Meti, USA) allows an anatomically correct demonstration of various physical and clinical signs including bleeding, breathing, blinking eyes, etc. and improves the fidelity of the simulations. The whole-body simulators are used mainly for the training of the physicians and other medical and technical stuff to work properly in different situations $[1,2]$.

ASL 5000 (Ingmar Medical, USA) is a lung simulator that is represented by a valve that is driven by the computer. The simulator provides possibility of setting airway resistance, alveolar compliance and other possibilities like different airway resistance for inspiratory and expiratory phase of the breathing cycle. The ASL 5000 provides wide range of analysis that can be used to calculate work of breathing and other parameters. The model also allows simulation of spontaneous breathing with adjustable power of breathing muscles. The simulator is used to study different ventilatory regimens $[3,4,5]$.

The aim of the study is to connect the whole-body simulator METI ECS and lung simulator ASL 5000 in order to combine the properties of both simulators. Main aim was to increase the fidelity of the simulation and number of features that can be controlled and monitored and minimize the interference of both simulators between each other. The connected simulators are used during the courses for the bachelor students at Faculty of biomedical engineering in Kladno in the study branch biomedical technician and for the students studying the subject respiratory therapy.

\section{METHODS}

First, we analyzed the properties of both systems independently and we designed the connection of both systems according to the analysis.

The ASL 5000 simulator is designed as a computer controlled valve moving within the cylinder. The alveolar compliance can be set in the wide range corresponding to the physiological values for healthy and diseased lung. The compliance is simulated according to (1):

$$
\mathrm{d} V=\mathrm{d} p . C,
$$

where $V$ represents volume, $p$ represents ventilatory pressure and $C$ is simulated compliance. Similarly, the airway resistance is simulated according to (2):

$$
\mathrm{d} V / \mathrm{d} t=R / \mathrm{d} p,
$$

where $R$ represents airway resistance and $t$ is time. Most of the parameters including airway resistance and alveolar compliance can be changed during the simulation and their effect can be observed. The simulator allows measuring of intrapulmonary parameters like alveolar pressure, tidal volume, etc. and analysis of measured signals.

The whole-body simulator METI ECS represents the model of the human. The simulator is constructed to be similar to the real anatomy of the human body. It consists of three basic parts: respiratory, cardiovascular and pharmacological models that are linked between each other in the similar way as in the live organism [6]. Therefore, it is possible to study the interaction between the systems and study the reaction of the cardiovascular system on the changes in the respiratory part of the simulator, e.g. blood gases can be analyzed after the changes in the ventilatory parameters. Most of the parameters that can be studied are simulated only mathematically in the software part of the simulator.

Both simulators were connected in the pneumatic part of the system (Fig. 1) and their interaction was minimized. The connection is realized in the abdomen part of the whole-body simulator. It allows use all parameters provided by both models for the education purposes.

The artificial ventilation of simulator ASL 5000 was analyzed for normal condition of the respiratory system and for asthma patient. The same simulations were repeated after 


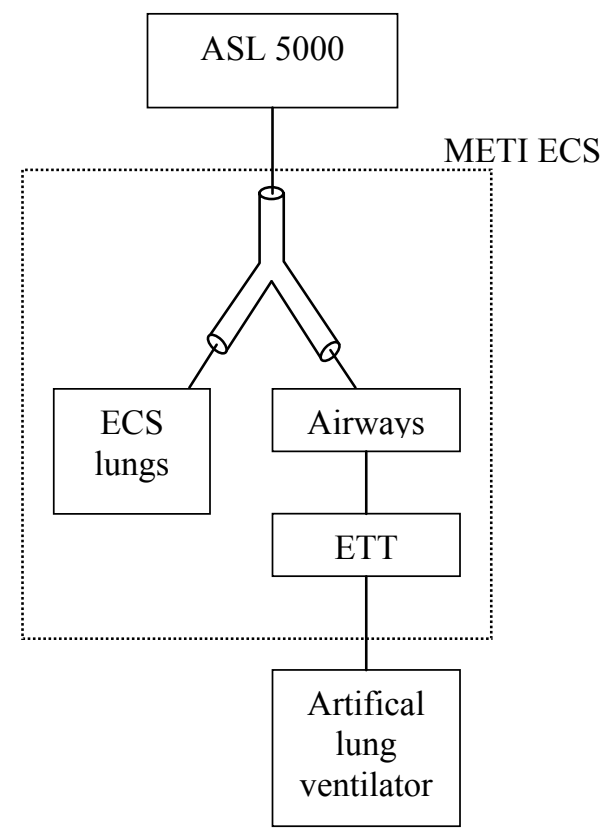

Fig. 1: The scheme of connection of simulators METI ECS and ASL 5000.

connection of both systems. The connection was optimized to minimize the interference of the simulators.

Artificial lung ventilator Veolar (Hamilton Medical, USA) was used to ventilate the simulators. The ventilator was used in the volume-controlled regimen with following ventilatory parameters: tidal volume $V_{t}=0.5 \mathrm{~L}$, breathing frequency $f=15$ bpm, zero end expiratory pressure PEEP $=0 \mathrm{cmH}_{2} \mathrm{O}$. All ventilatory parameters were held constant during the simulations with only the exception: tidal volume $V_{t}$ was increased to $1.0 \mathrm{~L}$ during the ventilation of both systems connected together.

\section{RESULTS}

Fig. 2 depicts time courses of the flow and pressure waves for simulation of normal lung mechanics of an adult human. The signals are saved from the circuit consisting of artificial lung ventilator and lung simulator ASL 5000. The airway resistance was set to $5 \mathrm{cmH}_{2} \mathrm{O} / \mathrm{l} / \mathrm{s}$ and alveolar compliance was set to $80 \mathrm{ml} / \mathrm{cmH}_{2} \mathrm{O}$.

Peak inspiratory pressure in the ventilatory circuit is slightly above $10 \mathrm{cmH}_{2} \mathrm{O}$ and peak flow is approximately 45 $\mathrm{lpm}$. Mean pressure in the circuit is approximately $6 \mathrm{cmH}_{2} \mathrm{O}$ and mean flow is around $12 \mathrm{lpm}$. Volume delivered to the lung is $500 \mathrm{ml}$.

Fig. 3 depicts same signals for the connection of the ventilator with both simulators: METI ECS and ASL 5000. Only the difference is in the flow occurred during inspiratory pause between both simulators. All other parameters including peak pressure and peak flow remain almost the same.

Airway resistance $25 \mathrm{cmH}_{2} \mathrm{O} / \mathrm{l} / \mathrm{s} /$ was used to simulate the patient with asthma, alveolar compliance was $80 \mathrm{ml} / \mathrm{cmH}_{2} \mathrm{O}$. The time trends of the intrapulmonary signals for the

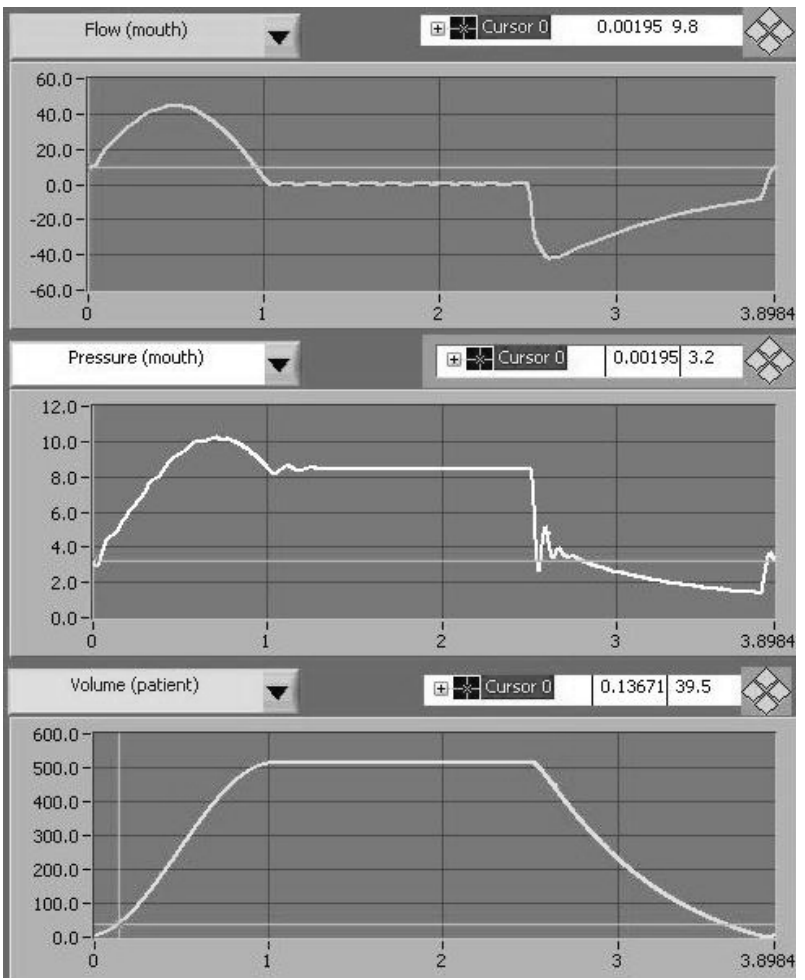

Fig. 2: The time courses of the flow at the mouth, pressure at the mouth and volume delivered to the lung for normal lung mechanics. Measurement was made for connection of the ventilator and simulator ASL 5000.

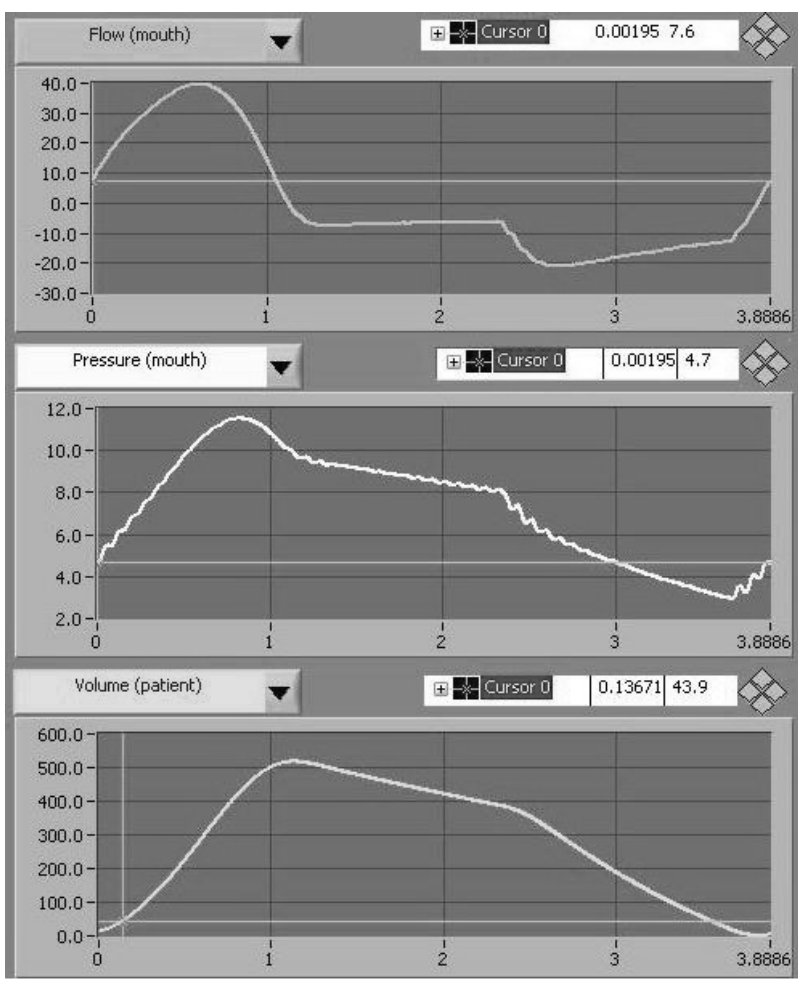

Fig. 3: The time courses of the flow at the mouth, pressure at the mouth and volume delivered to the lung for normal lung mechanics. Measurement was made for the connection of the ventilator and whole-body simulator METI ECS and lung simulator ASL 5000. 
connection of the ventilator and lung simulator ASL 5000 are depicted in Fig. 4. Peak inspiratory pressure is approximately $26 \mathrm{cmH}_{2} \mathrm{O}$ and peak flow in the ventilatory circuit is around 36 lpm. Mean airway pressure is around $12 \mathrm{cmH}_{2} \mathrm{O}$ and mean mean flow is approximately $12 \mathrm{lpm}$.

Very similar values and time trends of the pressure and flow waves are depicted in Fig. 5 for the connection of the ventilator with both simulators: METI ECS and ASL 5000.

\section{CONCLUSION}

The unique connection of two simulators was designed and realized. The connection of the systems allows usage of the advantages of both simulators. The connection was optimized and the values of the intrapulmonary parameters observed at connection of ventilator with both simulators were similar to that observed during the connection of the ventilator and simulator ASL 5000 only. It was necessary to set new value of dead space for ASL 5000 and increase oxygen consumption, decrease carbon dioxide production and shift $\mathrm{pH}$ because of higher tidal volume $\mathrm{V}_{\mathrm{t}}$ used during connection of both simulators.

Connection of both systems allows study wide range of parameters including effect of pulmonary mechanics upon the pressures and flows in the ventilatory circuit and also upon the blood gases.

Modern equipments used during the education increase an interest of the students during the exercises.

\section{ACKNOWLEDGMENT}

This research work has been supported by CTU SGS11/171/OHK4/3T/17.

\section{REFERENCES}

[1] A. Wilford, T. J. Doyle. Integrating simulation training into the nursing curriculum. Br J Nurs, vol. 15, pp. 926-30, 2006

[2] G. Lloyd, J. Kendall, S. Meek, P Younge. High-level simulators in emergency department education: thoughts from the trainers' perspective. Emerg Med J, vol. 24, pp. 288-291, 2007.

[3] R. L. Chatburn, "Which Ventilators and Modes Can Be Used to Deliver Noninvasive Ventilation?" Respiratory Care, vol. 54, pp. 85-101, January 2009.

[4] J. C. Ferreira, D. W. Chipman, N. S. Hill, R. M. Kacmarek. Bilevel vs ICU ventilators providing noninvasive ventilation: effect of system leaks: a COPD lung model comparison. Chest, vol. 136, pp.448-456, 2009

[5] J. Kattwinkel, C. Stewart, B. Walsh, M. Gurka, A. Paget-Brown. Responding to compliance changes in a lung model during manual ventilation: perhaps volume, rather than pressure, should be displayed. Pediatrics, vol. 123, pp. e465-470, 2009.

[6] W. L. Van Meurs, E. Nikkelen, M. L. Good, "Pharmacokineticpharmacodynamic model for educational simulations. "IEEE Trans Biomed Eng, vol. 45, pp. 582-590, 1998

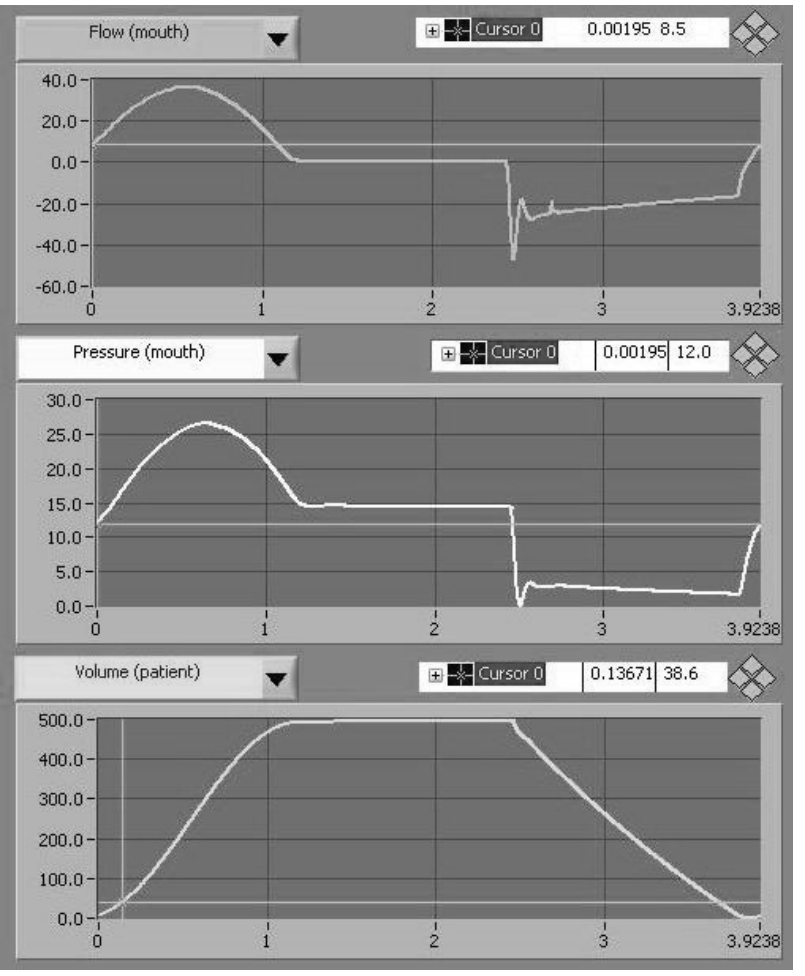

Fig. 4: The time courses of the flow at the mouth, pressure at the mouth and volume delivered to the lung for asthma patient. Measurement was made for connection of the ventilator and simulator ASL 5000.

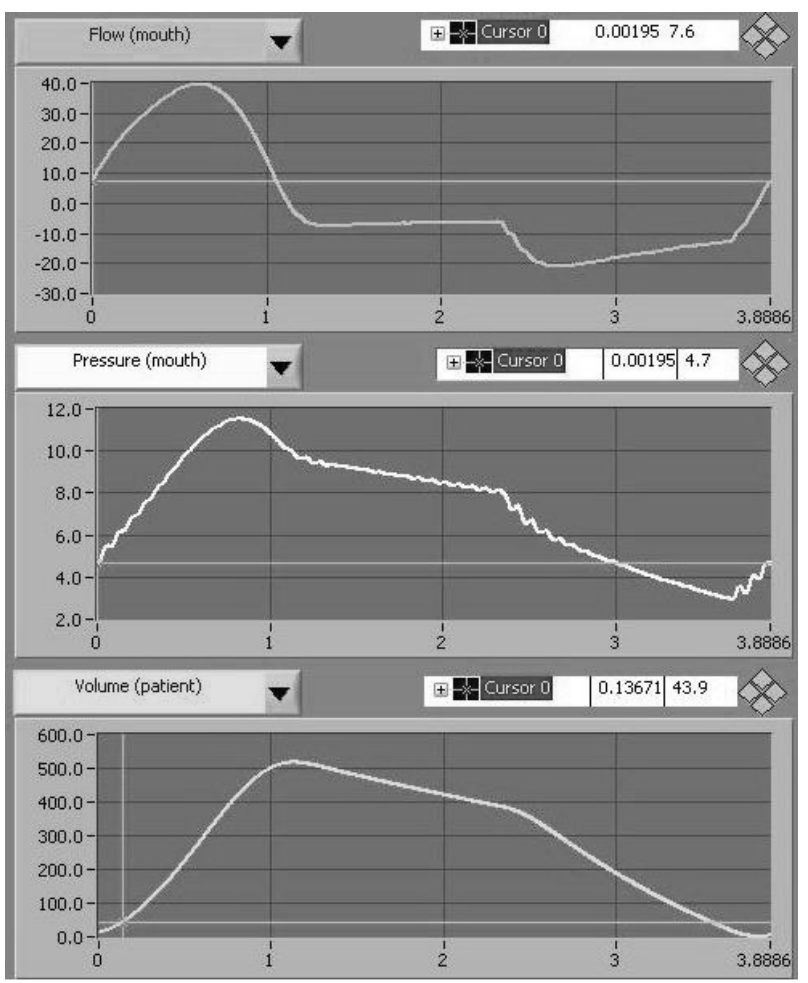

Fig. 5: The time courses of the flow at the mouth, pressure at the mouth and volume delivered to the lung for normal lung condition. Measurement was made for the connection of the ventilator and whole-body simulator METI ECS and lung simulator ASL 5000.

\section{Creative Commons Attribution License 4.0 (Attribution 4.0 International, CC BY 4.0)}

This article is published under the terms of the Creative Commons Attribution License 4.0

https://creativecommons.org/licenses/by/4.0/deed.en_US 